\title{
Atuaç̃̃o no Ensino Superior: Um novo Campo Para o Psicólogo Escolar
}

\author{
Maria Nasaré Fonseca Serpa ${ }^{1}$ \\ Acácia Aparecida Angeli dos Santos ${ }^{2}$
}

\section{Resumo}

\begin{abstract}
Este estudo teve como objetivo identificar a existência de estruturas de atendimento e orientação ao universitário; caracterizar os serviços e programas oferecidos; verificar a composição da equipe profissional; a existência e atuação do psicólogo escolar na equipe e avaliação dos serviços e programas. Um questionário foi utilizado para coleta de dados, sendo que dos 121 distribuídos, 61 foram respondidos. 80\% das IES que responderam, declaram possuir serviços específicos de orientação, que aparecem com frequiência significativamente maior nas IES comunitárias, seguidas das públicas e das privadas. O psicólogo escolar está presente em $75 \%$ dos serviços, e oferece diversos programas ao universitário. Tanto as IES que não possuem psicólogo na equipe, como as que não possuem serviços de orientação recorrem a encaminhamentos externos. A maioria das instituições entendem como necessária a criação de uma estrutura de apoio à clientela universitária.
\end{abstract}

Palavras-chave: Universitários; Ensino Superior; Atendimento ao Universitário; Psicólogo Escolar.

\section{Higher Education: a New Field for the School Psychologist}

\begin{abstract}
This study was performed with the following objectives: to identify the existence of Students Counselling and Orientation Services; to characterize the Services and programs offered; to verify the professional composition of the Service staff. Forms were distributed to the universities, asking questions about the presence and characteristics of the Counselling Services. Of the 121 forms distributed to the universities, 61 were returned. Eighty percent of these declare to have such services, being more frequent in the Communitary-type Institutions. In 75\% of the Services, a School Psychologist is available, and they offer several types of programs to the students. The alternative treatments to the problems of the students in this institutions where there is no Counselling Services or where the School Psychologist is not available were also discussed. The importance of a Counselling Service is recognized by the institutions that do not have one.

Key-words: undergraduates; Higher Education; Counseling Services; School PsychologistKey words: concept formation; conceptual development;
\end{abstract} theory view.

\section{INTRODUÇÃo}

\begin{abstract}
A Psicologia Educacional e a Psicologia Escolar, embora sejam consideradas áreas de intensa produtividade e que de longe suplantam todas as demais áreas da Psicologia no que se refere à elaboração de trabalhos e experimentos, ainda têm muito a evoluir, para atender à necessidade de uma ampla compreensão dos diversos fatores que interferem no processo de ensino e de aquisição do conhecimento, como aliás no desenvolvimento psico-social dos mais jovens A formação do Psicólogo Escolar, devido à sua relevância, tem sido objeto de vários estudos e pesquisas de âmbito nacio-
\end{abstract}

nal e internacional e a partir deles foram evidenciados os requisitos mínimos necessários à desejada capacitação profissional. (Tomanik,1992; Granja, 1995). No Brasil, vários estudiosos têm sido unânimes em demonstrar que tal a formação não tem sido adequada e conseqüentemente não têm consonância com a realidade educacional do país (Witter, 1987; Guzzo e Witter, 1987; Yaslle, 1990).

Oliveira (1991), ao descrever e analisar a formação do Psicólogo Escolar em Belém do Pará, verificou que a tendência curricular é privilegiar a for-

\footnotetext{
${ }^{1}$ Psicóloga, Mestre em Educação e Docente da Universidade São Francisco. E-mail: lezaserpa@uol.com.br

${ }^{2}$ Psicóloga, Doutora em Psicologia Escolar pela USP e Docente da Universidade São Francisco. E-mail: acaciasantos@terra.com.br
} 
mação do psicólogo clínico, com poucas disciplinas voltadas para a área de Psicologia Escolar. Benchaya (1992), em pesquisa realizada no mesmo local, demonstrou que a atuação de estagiários da área é basicamente remediativa, desvinculada da teoria e descontextualizada do processo educacional, servindo apenas para o cumprimento das exigências legais. Detectou, também, a concentração de conteúdo voltados para atuação apenas nos anos iniciais de escolarização.

Dados similares foram obtidos por Witter (1996), em seu estudo sobre o tema Psicologia Escolar: produção científica, formação e atuação, em periódicos nacionais e estrangeiros quando constatou que, mesmo fora do Brasil, a preocupação com a formação do psicólogo escolar visa predominantemente à atuação na pré-escola e no ensino fundamental, constatando sua falta de preparação para trabalhar com alunos que estejam em outros níveis de escolaridade, de modo especial no meio universitário.

Gonçalves (1994), tendo como objeto de estudo a formação e os estágios em Psicologia Escolar, verificou que a formação nessa área tem sido inadequada e que há uma estagnação curricular, demonstrada pelas poucas mudanças que têm ocorrido nos últimos anos. Outros estudos confirmam que o enfoque predominante dos cursos na área clínica limita a compreensão da problemática educacional numa perspectiva mais ampla, gerando dificuldades à prática profissional do Psicólogo Escolar, não permitindo que os modelos tradicionais de atuação sejam superados e fazendo que cresça a distância existente entre a demanda social e os serviços prestados à população (Jobin e Souza, 1996; Alves, 1997).

Estudiosos da área consideram que o Psicólogo Escolar deve ter uma formação que o instrumentalize para usar os recursos científicos da psicologia para o desenvolvimento dos indivíduos. Ressaltam que seus instrumentos, constituídos de conhecimento científico e técnicas específicas, devam contribuir, de modo cooperativo, na prevenção e solução de problemas no cotidiano escolar. Além disso, deve ter também um sólido embasamento teórico, pesquisa e prática em Psicologia e áreas afins (Masini,1981; Khouri e cols.,1984; Pfromm Netto, 1985).

Objetivando conhecer os tipos de atuação do psicólogo na educação, Maluf (1994) realizou uma pesquisa com alguns profissionais da área, reconhecidos por seus pares como de atuação inovadora, e concluiu que o curso de graduação não é suficiente para promover uma visão multidisciplinar da área educacional

Não resta dúvida que o Psicólogo Escolar tem sido pressionado a apresentar uma contribuição mais eficaz de atuação dentro da variedade de problemas escolares que se apresentam, especialmente no que se refere ao processo de aprendizagem. A prática mais abrangente e indireta, voltada para a estruturação de programas para alunos com dificuldade e a preparação e treinamento de professores, marca este momento como um grande salto da psicologia na visão de Almeida e Guzzo (1992). O foco de atenção centrada no aluno desloca-se e, embora conservando a intervenção basicamente remediativa, surge também um enfoque na instituição educacional, aliada à atuação de outros profissionais (Novaes, 1996).

Gradativamente, com o rompimento da visão reducionista da escola, oriundo dos movimentos educacionais, são reconhecidas as necessidades e vantagens de atitudes preventivas para o desenvolvimento psicológico dos educandos, o que tem levado a novos padrões na prestação de serviços na área (Conselho Federal de Psicologia, 1994). O Psicólogo Escolar deve então trabalhar aliado ao educador, integrando aprendizado e ensino aos conhecimentos pedagógicos e de desenvolvimento, valorizando as diferenças culturais, e, por fim, divulgando estes conhecimentos, para que ocorra o progresso da própria Psicologia Escolar (Jobim e Souza, 1996).

Von Buettner (1997) sugere entre outras metas, o desenvolvimento de práticas de atuação mais ousadas, ocupando temas e espaços pouco estudados como, por exemplo, o do ensino superior, expandindo-se os campos de atuação da disciplina, fazendo-se ao mesmo tempo, avaliação crítica dos resultados dos métodos dessa atuação.

Outros desafios ainda se agregam a esses, tendo em vista a extrema rapidez no avanço do conhecimento e o crescimento vertiginoso das inovações tecnológicas numa era de globalização da economia e da informação. Há uma exigência premente para que todos os profissionais reflitam sobre o alcance dessa metamorfose no campo educacional, produzindo propostas adaptadas a essa nova realidade.

Estudos sobre universitários têm apontado para existência de fenômenos, tais como a evasão e a reprovação, entre outros, que requerem atenção espe- 
cial dos estudiosos da psicologia e áreas afins. Fica evidente que uma instituição de ensino superior não pode contentar-se apenas com o desempenho acadêmico, a freqüência escolar e a formação profissional dos seus estudantes, tendo em vista que é de sua responsabilidade a formação integral do ser humano (Cabrera e cols., 1992; Polydoro, 1995 e 2001; Sbardelini, 1997, Almeida, Soares e Ferreira, 1999).

Muitos estudos têm indicado que o ensino superior constitui-se num campo de trabalho privilegiado para o Psicólogo Escolar, porque a ausência de um programa de apoio ao estudante contribui para a perpetuação de uma série de problemas de várias ordens, como uma formação básica insuficiente ou inadequada, falta de habilidades de leitura, escrita e estudo, insegurança nas tomadas de decisões, entre outros (Almeida, 1990; Witter, 1985; Santos, 1998). O psicólogo escolar deve ocupar esse espaço criado nas universidades, a partir da compreensão da importância do atendimento à sua clientela. Para que o psicólogo consiga ocupá-lo com eficiência é necessário repensar e reestruturar sua formação profissional, com a adoção de medidas curriculares que privilegiem a formação ampla e generalista, com atenção ao atendimento ao universitário e ao adulto, quesitos estes por vezes negligenciados atualmente, como demonstram as pesquisas realizadas por Benchaya (1992), Gonçalves (1994) e Witter (1996).

Como pode ser visto, a preocupação com o estudante universitário tem sido ampliada para além dos aspectos cognitivos, ressaltando-se a importância da busca de soluções para questões de adaptação à vida acadêmica e universitária do estudante, Tendo em vista a importância do componente emocional na vida humana, o que não pode ser desprezado pela universidade (Ellior e Witt, 1986; Felner e Adan, 1990; Luckes, 1992).

A partir da identificação dos fatores de risco ao sucesso acadêmico na opinião de 512 estudantes numa universidade católica do interior de São Paulo, Pacheco (1996) alerta para a necessidade de atenção aos alunos ingressantes em uma instituição de ensino superior, levando em conta aspectos pessoais, acadêmicos, familiares e institucionais e considera fundamental que as IES definam e implementem programas institucionais, que se caracterizarão num importante campo de atuação para o psicólogo escolar.
Confirmando tal importância, Serpa, Andraus e Joly (1997) realizaram um estudo de caracterização do usuário de um Serviço de Orientação ao Estudante (SOE) numa universidade comunitária e verificaram que os principais motivos apontados pelos alunos para a procura do serviço incluem problemas emocionais (angústia, ansiedade, depressão, indecisão, conflitos, insegurança, medo, solidão e timidez) e também a necessidade de ser ouvido e orientado.

A identificação da necessidade do preenchimento desse espaço de intervenção reforça alguns dos questionamentos que vêm há algum tempo merecendo a preocupação de vários estudiosos quanto à formação do psicólogo escolar, levando-os a constatar que é imprescindível que se desenvolvam estratégias preventivas frente às constantes e rápidas mudanças tecnológicas e sociais que estão a exigir da ciência, e especialmente da Psicologia, respostas para a solução dos problemas gerados por estas transformações.

Acredita-se que, a partir de estudos de levantamento e caracterização desses serviços e das formas de atuação dos psicólogos, seja possível obter-se subsídios que forneçam às instituições de ensino formas alternativas para a implantação e desenvolvimento de programas preventivos para seus estudantes, que favoreçam o processo de desenvolvimento pessoal e profissional integrado e harmonizado.

Considerando-se a escassez de dados sobre a estrutura e o funcionamento dos programas/serviços de orientação existentes nas IES brasileiras, bem como informações sobre a presença e a atuação de psicólogo escolar nesses serviços, este estudo foi proposto com os seguintes objetivos:

1- Verificar a composição da equipe técnica nos serviços existentes, a existência do Psicólogo Escolar como integrante da equipe; e os programas/serviços oferecidos por esse profissional;

2- Identificar os programas ou atividades desenvolvidas, formas de divulgação e demanda;

3- Verificar os procedimentos utilizados pelas IES que possuem Serviços de Atendimento ao Universitário, mas que não contam com um Psicólogo Escolar na equipe, para o encaminhamento dos problemas que seriam atendidos por esse profissional;

4- Verificar os procedimentos utilizados pelas IES que não possuem Serviços de Atendimento ao Universitário, para o encaminhamento dos problemas enfrentados pelo corpo discente e a posição destas sobre as perspectivas de implantação desse tipo de serviço. 


\section{MÉT0D0}

Trata-se de um estudo descritivo, pelo qual procurou-se obter informações sobre prestação de serviços de atendimento ao universitário em Instituições de Ensino Superior (IES) no Brasil, a presença e formas de atuação de Psicólogos Escolares nesses serviços; bem como os tipos de programas por eles desenvolvidos.

\section{Amostra}

A pesquisa foi realizada em 61 Instituições de Ensino Superior (IES) brasileiras, que responderam ao questionário enviado, correspondendo a 50,5\% das 121 instituições contatadas. As IES que compõem o grupo de informantes, foram classificadas em dois grupos 1) com Serviço de Atendimento ao Universitário e 2) sem Serviço de Atendimento ao Universitário e caracterizadas segundo a sua natureza: pública, privada e comunitária.

Definiu-se como escolas públicas as criadas ou incorporadas, mantidas e administradas pelo Poder Público; privadas, as mantidas e administradas por pessoas físicas ou jurídicas de direito privado e comunitárias, as que são instituídas por grupos de pessoas físicas ou por uma ou mais pessoas jurídicas, inclusive cooperativas de professores e alunos que incluam na sua entidade mantenedora representantes da comunidade (LDB, p. 25). emergentes e sua opinião a respeito da necessidade de implantação de serviços de atendimento e uma questão relacionada à identificação do informante.

\section{Procedimento:}

A maioria dos questionários informativos foram entregues, em mãos, a representantes das IES e 28 questionários foram enviados por correio.

\section{Resultados E Discussão}

Como parte de um estudo mais amplo, apresentase aqui os resultados, bem como a análise e discussão das questões direta ou indiretamente relacionadas com a atuação do Psicólogo Escolar nos Serviços de Atendimento ao Universitário (SAU). A comparação de proporções das amostras foi realizada através do teste do qui-quadrado, com 1 ou 2 graus de liberdade de acordo com o tipo de tabela ( 2 X 2 ou 2 X 3$)$.

A Tabela 1 mostra a distribuição da presença ou não de serviços de atendimento ao universitário (SAU), segundo o tipo de IES, seguindo a classificação entre Públicas, Privadas ou Comunitárias.

Considerando a existência ou não do SAU, constata-se que a maioria $(80,3 \%)$ das IES que respondem

Tabela 1: Divisão das IES segundo a presença do Serviço de Atendimento ao Universitário (SAU)

\begin{tabular}{lcccccc}
\hline Tipos de IES & com SAU & $\%$ & Sem SAU & $\%$ & TOTAL & $\%$ \\
\hline Pública & 32 & $84,2 \%$ & 6 & $15,8 \%$ & 38 & $100 \%$ \\
Privada & 5 & $50,0 \%$ & 5 & $50,0 \%$ & 10 & $100 \%$ \\
Comunitária & 12 & $92,3 \%$ & 1 & $7,7 \%$ & 13 & $100 \%$ \\
\hline Total & 49 & $80,3 \%$ & 12 & $19,7 \%$ & 61 & $100 \%$ \\
\hline $\mathrm{c}^{2}{ }_{(2)}=7,363 ; p<0,05$ & & & & & &
\end{tabular}

\section{Instrumento}

Questionário informativo, constituído de 21 questões abertas, fechadas ou mistas, com 6 questões visando a identificação e caracterização das universidades estudadas; 8 questões abrangendo informações sobre a existência, o funcionamento e a estratégia usada para a divulgação dos serviços de atendimento ao universitário; 3 questões destinadas apenas aos psicólogos que atuam nos serviços, referentes aos tipos de programas/ serviços por eles desenvolvidos e 3 questões referindose à postura do informante frente às necessidades ao questionário ( $\mathrm{N}=61)$, possui algum tipo de serviço destinado a este fim. Entre as IES do tipo pública $(\mathrm{N}=38)$, verifica-se que $84,2 \%$ possuem algum tipo de serviço; nas do tipo comunitária ( $\mathrm{N}=13)$, o SAU está presente em $92,3 \%$ e nas do tipo Privada $(\mathrm{n}=10)$, há $50 \%$ que possuem SAU. Assim verifica-se a existência de diferença significativa entre o tipo de IES e a presença do SAU $\left(\mathrm{c}_{(2)}^{2}=7.363 ; \mathrm{P}<0,05\right)$, apontando para o que pode ser considerado como uma maior preocupação com a atendimento das necessidades do alunado nas IES comunitárias. 
As análises e discussões apresentadas a seguir referem-se apenas às IES que possuem SAU $(\mathrm{N}=49)$. Com relação à questão de que tipo de população é atendida pelo serviço, constatou-se que, além dos alunos de graduação obviamente atendidos por todas, 50,0\% das Instituições atendem aos alunos da pós-graduação, $54,3 \%$ prestam serviços à família e 41,3\% à comunidade, sendo computados nesta última categoria atendimentos aos professores, funcionários e à comunidade externa. Os dados sugerem que o aluno de pós-graduação ainda não foi compreendido como integrante da comunidade universitária, o que pode ter raízes na visão da graduação como estágio terminal da vida universitária, situação ainda freqüiente no Brasil (Witter, 1985).

Ao se observar a abrangência de atendimento dos Serviços de Atendimento ao Universitário, verificase que sua extensão de apoio à comunidade é de grande importância, pois a Universidade deve ter em mente que sua clientela não se limita aos alunos, mas engloba também a família e a comunidade em que se insere e que a mantém (Mezomo, 1994, Pacheco, 1996). O próprio enfrentamento da crise da Universidade pressupõe, segundo Dreze e Debele (1983), a participação comunitária e a permeabilidade entre o meio universitário e o não-universitário.

As Tabelas 2 e 3, que se seguem, mostram as áreas de atenção do atendimento prestado e a constituição da equipe de profissionais envolvidos no atendimento.

Verifica-se que o atendimento é oferecido com a mesma frequiência nas áreas "Psicológica" e "Social" com 73,9\%, seguidas das áreas "Educacional" com 47,8\%, "Saúde" com 41,3\% e "Outras" com 10,9\%. $\mathrm{Na}$ categoria "Saúde" foram reunidas as respostas referentes aos seguintes serviços: médico, psiquiátrico, terapia ocupacional, odontológico, enfermagem, nutrição, clínica geral, clínica do trabalho e primeiros socorros, enquanto na categoria "Outras" foram citados: bem-estar espiritual, assistência jurídica, computação, estrutura acadêmica, esporte e lazer. A compreensão do aluno como inserido em diversos contextos que não se limitam ao meio universitário exige que também o atendimento prestado a este seja feito de forma pluralista. Como nenhuma área consegue isoladamente suprir a todas as demandas, vale lembrar a importância de ser desenvolvida uma atitude de interdisciplinaridade, conforme defendido por Von Buettner (1997).

Os dados apresentados na Tabela 3 permitem verificar a presença do psicólogo na composição da equipe profissional dos Serviços de Atendimento ao Universitário. Observa-se que das 49 instituições que relatam possuir o serviço, $09(18,3 \%)$ não responderam à esta questão, e para o espaço amostral serão consideradas as 40 IES que forneceram tais dados. Destas, $34(85 \%)$ referem-se ao oferecimento de "Atendimento

Tabela 2 - Distribuição das áreas de atendimento existentes no SAU

\begin{tabular}{lll}
\hline Área & $\mathrm{N}$ & $\%$ \\
\hline Psicológica & 34 & $73,9 \%$ \\
Social & 34 & $73,9 \%$ \\
Educacional & 22 & $47,8 \%$ \\
Saúde & 19 & $41,3 \%$ \\
Outras & 5 & $10,9 \%$ \\
\hline
\end{tabular}

Nota: 3 IES, que correspondem a 6,1\% da amostra não responderam à esta questão.

Tabela 3 - Composição profissional do SAU

\begin{tabular}{lll}
\hline Categoria profissional & $\mathrm{N}$ & $\%$ \\
\hline Psicólogo & 31 & 77,5 \\
Assistente Social & 28 & 70,0 \\
Pedagogo & 14 & 35,0 \\
Psiquiatra & 7 & 17,5 \\
Outros & 9 & 22,5 \\
\hline
\end{tabular}

Nota: 9 IES, que correspondem a 18,03\% da amostra não responderam à esta questão. 
Psicológico", muito embora 03 IES não relatem a presença de psicólogo, deixando dúbio que tipo de atendimento seria este. Em 31 IES (77,5\%) o psicólogo está presente como parte constituinte da equipe. $\mathrm{Ou}-$ tra categoria profissional que merece destaque na análise é a dos assistentes sociais, já que estes figuram do quadro de atendimento em $70 \%(\mathrm{n}=28)$ da amostra. Em menor freqüência aparecem pedagogos e psiquiatras. Também foram citados esporadicamente: professores, sacerdotes, terapeutas ocupacionais, dentistas, nutricionistas, médicos, advogados, sociólogos, operadores de computador e técnicos administrativos.

Quatro serviços relatam ainda a existência de "estagiários de psicologia". Nota-se pela descrição acima, que o psicólogo é figura freqüente na composição do quadro de efetivos dos Serviços de Atendimento ao Universitário, confirmando a real necessidade de sua presença, coerente com a afirmação de Luckesi (1992) sobre a importância do componente emocional na adaptação à vida acadêmica e com dados de estudo anterior destas autoras, que constataram que as questões de fundo emocional estão entre os principais motivos de procura de serviços de apoio pelos universitários (Serpa e Santos,1997).

Outro resultado interessante foi obtido, ao se investigar como o psicólogo trabalha junto com os outros profissionais alocados nos serviços. Em 60,6\% dos casos o trabalho do psicólogo é desenvolvido tanto de forma individual como em equipe; $21,2 \%$ desenvolvem trabalhos só em equipe e 18,2\% relatam trabalhos apenas individualmente. Nota-se que apesar da já frisada importância da cooperação interdisciplinar, em alguns serviços o psicólogo atua de maneira desvinculada ou independente da equipe; ainda em alguns casos o psicólogo é membro solitário e único no atendimento. Os dados obtidos evidenciam um dos atuais desafios à atuação do psicólogo, que exige uma visão transdisciplinar lúcida para evitar o isolamento profissional (Maluf, 1994; Novaes, 1996).

Na Tabela 4 aborda-se os serviços e programas em que há participação do psicólogo. Foi permitido assinalar mais de uma alternativa, portanto a soma das porcentagens ultrapassa $100 \%$.

O psicólogo pode desenvolver vários programas e serviços dentro do SAU. Devido à esta variedade de possibilidades de atuação, a formação generalista deste profissional é desejável e deve ser encorajada para que este importante campo de trabalho não fique desassistido ou tenha atendimento incompleto. Esta formação, defendida por Witter (1985), permitiria ao profissional atuar em todas as áreas onde fosse requerido. A formação generalista deve ser complementada pela especialização, e não ser substituída por esta. Novaes (1996) ao abordar os desafios da psicologia escolar para o terceiro milênio, ressalta a importância da visão transdisciplinar na formação psicológica. Pela análise dos dados aqui obtidos, constata-se que a grande maioria $(96,8 \%)$, oferece programa de orientação individual; a seguir surgem encaminhamentos internos e externos com a mesma frequiência de $87,1 \%$; também são freqüentes o acompanhamento individual $(77,4 \%)$, a orientação de grupos $(58,1 \%)$, atendimento à família $(58,1 \%)$ e programas especiais $(58,1 \%)$. Além destes, vários outros tipos de serviços foram citados, denotando a variedade da atuação psicológica neste campo.

Uma vez discutida a importância do Serviço de Atendimento ao Universitário no encaminhamento das dificuldades inerentes ao aluno, bem como a necessidade do psicólogo na estruturação e funcionamento do mesmo, obteve-se dados sobre as IES que não dispõem de psicólogos nos serviços oferecidos à clientela universitária $(\mathrm{n}=15)$, e sobre aquelas que não possuem Serviços de Atendimento ao Universitário $(\mathrm{n}=12)$.

Em ambos os casos verifica-se que as instituições desprovidas de serviços específicos para este fim buscam rotas e métodos alternativos no encaminhamento das dificuldades. O coordenador do curso é predominantemente o profissional mais procurado para lidar com esses alunos; a diretoria, professores com horas para atendimento, as pró-reitorias e o encaminhamento externo são outras soluções encontradas pelas IES. Luckesi (1992) atenta para o despreparo com que muitas vezes a Universidade enfrenta os fatores ligados às dificuldades pessoais ou sociais de seus alunos.

Procurou-se também investigar junto às IES qual a postura que tinham frente à criação de um Serviço de Atendimento ao Universitário e o resultado obtido demonstrou que a maioria o considera importante e pretende criá-lo em curto prazo $(41,7 \%)$; em segundo lugar aparecem as que o consideram importante e têm planos de implantação em médio prazo $(33,3 \%)$; outras atribuem-lhe importância mas não têm planos para sua implantação $(16,7 \%)$ e poucas o consideram desnecessário $(8,3 \%)$. Nota-se que, exceção feita à pos- 
Tabela 4 - Programas Desenvolvidos pelo Psicólogo

\begin{tabular}{lll}
\hline Tipos de programas & no de IES & $\%$ \\
\hline Orientação individual & 30 & $96,8 \%$ \\
Psicoterapia breve individual & 16 & $51,6 \%$ \\
Acompanhamento individual & 24 & $77,4 \%$ \\
Orientação em grupo & 18 & $58.1 \%$ \\
Terapia em grupo & 6 & $19,4 \%$ \\
Acompanhamento em grupo & 6 & $19,4 \%$ \\
Atendimento à família & 18 & $58,1 \%$ \\
Encaminhamento fora da universidade & 27 & $87,1 \%$ \\
Encaminhamento dentro da universidade & 27 & $87,1 \%$ \\
Cursos & 10 & $32,3 \%$ \\
Palestras & 15 & $48,4 \%$ \\
Programas especiais (coord. Prof. E Chefias de depto) & 18 & $58,1 \%$ \\
Atendimento à comunidade & 7 & $22,6 \%$ \\
Orientação vocacional & 5 & $16,1 \%$ \\
Outros & 3 & $9,7 \%$ \\
\hline
\end{tabular}

Nota: 3 IES, que correspondem a 6,5\% da amostra não responderam à questão.

tura isolada de uma instituição, há consenso quanto à compreensão do Serviço de Atendimento ao Universitário como importante, inclusive com a maioria delas $(75,0 \%)$ planejando sua instalação em médio ou curto prazo.

\section{CONSIDERAÇões FinaIS}

A contribuição da Psicologia Educacional é de relevante importância como uma das alternativas de soluções para os problemas a que estão submetidas as IES. Sendo a área de maior produção científica da psicologia aplicada, poderá contribuir para o desenvolvimento de estratégias preventivas frente aos problemas até há pouco impensáveis.

A resposta às novas exigências citadas tem como fator aliado, senão obrigatório, a criação de programas e serviços de orientação destinados à população universitária. O Serviço de Atendimento ao Universitário representa um modelo muito adequado na organização e otimização deste atendimento, permitindo abordagem de diferentes problemas com tratamento interdisciplinar.

Existe no ambiente universitário uma procura por orientação e acompanhamento psicológico que não pode ser ignorada, mas deve ser suprida a contento, por ser fórmula reconhecidamente eficaz na melhora do apro- veitamento acadêmico e na formação integral do indivíduo não apenas como profissional, mas também como cidadão inserido na sociedade. Além disso, a mobilização interna provocada pelo conteúdo de alguns cursos, em especial em cursos como o de Medicina e Psicologia, deve ser contemplada na elaboração de serviços/programas de atendimento ao universitário.

O psicólogo escolar deve ocupar esse espaço criado nas Universidades, a partir da compreensão da importância do atendimento à sua clientela . Para que o psicólogo consiga ocupá-lo com eficiência é necessário repensar e reestruturar a formação deste profissional, com a adoção de medidas curriculares que privilegiem a formação ampla e generalista, com atenção ao atendimento ao universitário e ao adulto, quesitos estes por vezes negligenciados.

Já que os problemas inerentes ao ser humano geram reflexos no processo de ensino e aprendizado, e tendo conhecimento da diversidade desses, não se pode pensar na abordagem da orientação ao aluno sendo praticada por um profissional ou uma área do conhecimento de maneira isolada. A equipe de atendimento deve ter como requisito obrigatório a constituição multi e interdisciplinar.

Os programas/serviços implementados nas IES devem ser avaliados pela população usuária a fim de adequar-se à sua realidade e às suas necessidades. É extremamente importante que esses serviços considerem as habilidades básicas trazidas pelos ingressantes, 
que muitas vezes estão comprometidas pelas carências do ensino médio e pelos problemas socioeconômicos que obrigam o universitário ao desempenho da pesada carga de estudar e trabalhar em tempo integral, tal como têm sido retratados em vários estudos (Castanho, 1989; Cardoso, 1994; Carelli e Santos, 1998).

Para assegurar melhor aproveitamento das atividades desenvolvidas pelo SAU, é necessário garantir uma boa comunicação entre o serviço e a população a que está destinado, utilizando de todos os recursos disponíveis para a sua divulgação, fazendo-se

\section{REFERÊNCIAS}

Almeida, L. S. \& Guzzo, R. S. L. (1992). A relação psicologia e educação: perspectiva histórica do seu âmbito e evolução. Estudos de Psicologia, 9 (3), 117-131.

Almeida, L. S., Soares, A. P. C. E. \& Ferreira, J.A. (1999). Adaptação, rendimento e desenvolvimento dos estudantes de ensino superior. Construção/Validação do Questionário de Vivências Acadêmicas. Relatório Técnico da Investigação, Centro de Estudos em Educação e Psicologia, Universidade do Minho, Braga-PT.

Almeida, S. F. C. de (1990). Programa de apoio psicopedagógico a estudantes universitários (PAPEU/ UFC). Boletim da Associação Brasileira de Psicopedagogia, 9 (18), 97-106.

Alves, C. F. (1997). A formação do psicólogo para o trabalho emeducação: uma análise do curso de psicologia da PUC/ SP. Dissertação de Mestrado. PUC - São Paulo.

Benchaya, R. (1992). Percepção do estágio supervisionado em psicologia escolar: relato de estagiários e supervisores. Dissertação de mestrado, IPUSP. SP.

Cabrera, A. F.; Castañeda, M. B.; Nora, A. \& Hengstler, D. (1992). The covergence between two theories of college persistence. Journal of Higher Education, 63 (2)143-164.

Cardoso, S. M. V. (1994). A prática docente no ensino superior particular noturno - um estudo de caso (Tese de Doutorado). Faculdade de Educação, UNICAMP, Campinas. necessário também avaliar a eficácia das estratégias que estão sendo utilizadas para esse fim. Sugere-se como proposta final que novos estudos sejam conduzidos para constante avaliação e aprimoramento desta área que reúne importante campo de inserção do psicólogo com uma estrutura de atendimento adequado à comunidade universitária. A compreensão e reconhecimento desta importância contribuirão de maneira positiva na melhoria da formação acadêmica, no desenvolvimento da instituição universitária e mesmo para a necessária transformação da sociedade.

Carelli, M. J. G. \& Santos (1998). Condições temporais e pessoais de estudo em Universitários. Psicologia Escolar e Educacional, 2 (3), 265-278.

Castanho, M. E. (1989). Universidade à noite: fim ou começo de jornada? Campinas: Papirus, 128p.

Conselho Federal de Psicologia-CFP (1994). Psicólogo brasileiro: práticas emergentes e desafios para a formação. São Paulo: Casa do Psicólogo.

Dreze, J. E Debelle, J. (1983). Concepções de Universidade. Fortaleza: Edições Universidade Federal do Ceará.

Elliot, S. E Witt, J. (1986). The delivery of psychological service in schools: concepts processes and issues. London: Hillsdale.

Felner, R. D. E Adan, A. M. (1990). The school transitional environment project: an ecological intervention and evaluation, In: R. H. Price; E. Cowen; R. P. Lorion \& M. Ramos. Fourteen ounces of primary prevention. Washington: American Psychology Association, 111112 .

Gonçalves, C. L. C. (1994) Informação e estágio acadêmico em psicologia escolar no Brasil: análise curricular (Dissertação de Mestrado). PUC-Campinas.

Granja, E. C. (1995). Produção científica: dissertações e teses do IPUSP (1989-1980) (Tese de Doutorado). Instituto de Psicologia da USP. 
Guzzo, R. S. L. E Witter, G. P. (1987). A relação psicólogo-escola pública na região de Campinas: um estudo exploratório da opinião de diretores, Estudos de Psicologia, 5(1), 17-34.

Jobim E Souza, S. (1996). O psicólogo na educação: identidade e transformação. Coletâneas da ANPEPP, 7, 3745 .

Khouri, Y. G. e cols. (1984). Psicologia Escolar. São Paulo: EPU.

Luckesi, C. C. (1992). Educação universitária e a formação do ser humano. Revista da FAEEBA, 1(1), 31-35.

Maluf, M. R. (1994). Formação e atuação do Psicólogo na Educação: dinâmica de transformação. In: R. Archar (Coord.). Psicólogo Brasileiro: práticas emergentes e desafios para a formação. São Paulo: Casa do Psicólogo.

Masini, E. F. S. (1981). A ação da psicologia na escola. São Paulo: Moraes.

Mezomo, J. C. (1994). A qualidade na escola: falando a mesma linguagem. Universidade: a busca da qualidade, IBRAQS, 1(5), 256-263.

Novaes, M. H. (1996). Perspectivas da psicologia escolar no Brasil face às perplexidades no novo século. Conferência proferida no III Congresso Nacional de Psicologia Escolar e Educacional, Rio de Janeiro,18-22 de novembro, 1996.

Oliveira, F. de A. F. (1991). Área de atenção na formação do Psicólogo Escolar (mimeorg.).

Pacheco, E. M. C (1996). Indicadores de Risco de sucesso acadêmico segundo universitários. (Dissertação de Mestrado). PUC-Campinas.

Pfromm Netto, S. (1985). Psicologia: introdução e guia de estudo. São Paulo: Edusa.

Polydoro, S. A. J. (1995). Evasão em uma instituição de ensino superior: desafio para a Psicologia Escolar (Dissertação de Mestrado). PUC- Campinas.

Polydoro, S.A.J. (2001). O trancamento de matrícula na trajetória acadêmica do Universitário: condições de saída e de retorno à instituição (Tese de Doutorado). FE- UNICAMP.

Santos, A. A. A. (1998). Psicopedagogia no $3^{\circ}$ grau: avaliação de um programa de remediação em leitura e estudo. Proposições, 8, 1 (22), 27-37.

Sbardelini, E.T.B (1997). A Reopção de Curso na Universidade Federal do Paraná (Tese de Doutorado). Ribeirão Preto, SP: FMRP- USP.

Serpa, M. N. F.; Andraus, S. Jr. \& Joly, M. C. A. (1997) Student's guidance service: study of usuary's profile. Memorias In Congreso Regional de Psicología para Profisionales en América, Cuidad de México, p. 356.

Serpa, M. N. F. \& Santos, A. A. A. (1997). Implantação e primeiro ano de funcionamento do Serviço de Orientação ao Estudante-SOE, trabalho apresentado no XI Seminário Nacional das Universidades Brasileiras (SENUBRAS), out./97 Guarulhos.

Tomanik, E. A. (1992). Ser ou não ser: a pesquisa em psicologia no Brasil e a questão da cientificidade (Tese de Doutorado). PUC-São Paulo.

Von Buettner, G. E. B. P. (1997). Para melhorar a Psicologia Escolar... algumas sugestões... Informativo ABRAPEE, 6(3),5.

Witter, C. (1996). Psicologia Escolar: Produção científica, formação e atuação (1990-1994). (Tese de Doutorado). IP-USP.

Witter, G.P. (1985). O psicopedagogo no ensino superior: Um campo a ser conquistado. Boletim da Associação Estadual de Psicopedagogos de São Paulo, 4 (8), 5-10.

Witter, G. P. (1987). Quem é o Psicólogo Escolar? sua atuação prática. Anais da XVII Reunião Anual de Psicologia. USP-RP. Ribeirão Preto.

Yaslle, E. G. (1990). A formação do Psicólogo Escolar no Estado de São Paulo: subsídios para uma ação necessária (Tese de Doutorado). PUC-São Paulo.

Recebido em: 05/06/2001

Revisado em: 11/09/2001

Aprovado em: 11/12/2001 